CLINICAL STUDY

\title{
Plasma levels of free metanephrines and 3-methoxytyramine indicate a higher number of biochemically active HNPGL than 24-h urinary excretion rates of catecholamines and metabolites
}

\author{
N van Duinen, E P M Corssmit, W H A de Jong ${ }^{1}$, D Brookman, I P Kema ${ }^{1}$ and J A Romijn \\ Department of Endocrinology and Metabolic Diseases, Leiden University Medical Center, PO Box 9600, 2300 RC Leiden, The Netherlands and \\ ${ }^{1}$ Department of Clinical Chemistry, University Medical Center Groningen, University of Groningen, Groningen, The Netherlands \\ (Correspondence should be addressed to N van Duinen; Email: n.van_duinen@lumc.nl)
}

\begin{abstract}
Context: A substantial number of patients with head and neck paragangliomas (HNPGLs) have biochemically active tumors, evidenced by increased urinary excretion of catecholamines and metabolites, including 3-methoxytyramine (3MT). It is unclear whether plasma levels of these parameters are more sensitive to detect biochemical activity in HNPGL patients than urinary excretion rates.

Objective: To compare plasma free levels vs urinary excretion rates of deconjugated 3MT and combined metanephrines (MNs) in patients with HNPGL.

Patients and methods: We included 124 consecutive patients with HNPGL for screening of catecholamine excess by measurement of 24-h urinary excretion rates of deconjugated (nor)metanephrine, (nor)epinephrine, dopamine, vanillylmandelic acid, 3MT, and plasma free levels of (nor)metanephrine and 3MT.

Results: Plasma free 3MT levels were increased in 35 of the 124 patients (28\%), whereas 24-h urinary excretion of deconjugated 3MT was increased in 30 patients $(24 \%)(P=0.13)$. Plasma free MN levels were increased in seven patients $(6 \%)$ and urinary deconjugated $\mathrm{MN}$ levels in six patients $(5 \%)(P=1.00)$. Plasma free normetanephrine (NMN) levels were increased in seven patients $(6 \%)$, and five patients had increased urinary excretion of deconjugated NMN $(4 \%)(P=0.69)$. Plasma free combined MN levels (NMN, MN, and 3MT) were increased in 41 patients (33\%), whereas 24-h urinary excretion rates of deconjugated combined MNs were increased in 33 patients $(27 \%, P<0.05)$.

Conclusions: The combined levels of free MNs and free 3MT in plasma indicate a higher number of biochemically active HNPGLs than the 24-h urinary excretion rates of these markers.
\end{abstract}

European Journal of Endocrinology 169 377-382

\section{Introduction}

Head and neck paragangliomas (HNPGLs) are rare neuroendocrine tumors derived from parasympathetic ganglia (1). Some patients with HNPGLs have biochemically active tumors, evidenced by increased urinary excretion rates of catecholamines and their metabolites $(2,3,4)$. The majority of those patients have increased urinary excretion rates of 3-methoxytyramine (3MT), the 3-O-methylated metabolite of dopamine (3).

It is presently unknown whether plasma free concentrations of 3-O-methylated metabolites of catecholamines, including 3MT, are more sensitive parameters of biochemical activity of HNPGL than urinary excretion rates of catecholamines or deconjugated 3-O-methylated metabolites. For the diagnosis of pheochromocytoma, the measurement of plasma free metanephrine $(\mathrm{MN})$ concentrations is the optimal biochemical test with the highest sensitivity and specificity $(5,6,7,8)$. Therefore, the aim of this study was to assess whether plasma levels of free MNs and $3 \mathrm{MT}$ are more sensitive parameters of biochemical activity of HNPGL than urinary excretion rates of free catecholamines and their deconjugated metabolites.

\section{Subjects and methods}

We performed a cross-sectional study of 130 consecutive patients with HNPGL who were followed at the outpatient clinic of the Leiden University Medical Center, a tertiary referral center for patients with paragangliomas. For this purpose, clinical, biochemical, and radiological data of all consecutive patients with HNPGL were evaluated. All patients were investigated at the outpatient clinic according to structured standard clinical protocols. These included questions focused on tumorand catecholamine-related signs and symptoms, measurement of blood pressure in the supine position, and after $5 \mathrm{~min}$ in the upright position, in order to screen 
for orthostatic hypotension. In all patients, head and neck magnetic resonance imaging (MRI) was performed or had been performed within the previous 2 years.

Urine was collected during $24 \mathrm{~h}$ in duplicate under strict dietary regulations (patients abstained from pineapple, avocado, bananas, kiwi, nuts, plums, coffee, tea, and other caffeine-containing beverages) and after withdrawal of medication for at least 1 week or after changing antihypertensive medication to doxazosin for several weeks. In order to ascertain adequacy of urinary collection, 24-h urinary creatinine excretion rates were also measured. Blood samples were drawn after the second day of urine collection in the post-absorptive state. Blood samples were drawn from an i.v. catheter inserted into a forearm vein after 30 min of rest in the supine position and collected in cold, glutathionecontaining vacutainers. All blood samples were centrifuged immediately at $3000 \mathrm{rpm}$ for $10 \mathrm{~min}$ at $4{ }^{\circ} \mathrm{C}$. Plasma samples were stored at $-80{ }^{\circ} \mathrm{C}$ until analyses.

Biochemical screening included the measurement of urinary excretion rates of deconjugated (nor)metanephrine, free (nor)epinephrine, dopamine, and deconjugated 3MT excretion in two 24-h urinary samples and the measurement of plasma free (nor)metanephrines and 3MT concentrations.

In case of excessive catecholamine or metabolite excretion (i.e. any value above the upper reference limit in two urine samples or in the plasma sample), radiological assessment by 123I-metaiodobenzylguanidine (MIBG) scans and MRI and/or CT scans of thorax and abdomen were performed to identify the source of excessive catecholamine production. All patients with documented paragangliomas in the abdomen or thorax were excluded in this study because the interpretation of the biochemical results in relation to HNPGL could be confounded by the presence of these other paragangliomas.

We performed screening for succinate dehydrogenase (SDH) mutations in those HNPGL patients, who agreed upon genetic testing. Hereditary disease was diagnosed if mutations in the SDHB, SDHC, or SDHD genes were documented in the HNPGL patient and/or a family member.

We screened 130 consecutive HNPGL patients for catecholamine excess. Four patients with catecholamine excess were excluded from the current analyses because no additional radiological assessment was performed to exclude the presence of other paragangliomas not related to the head and neck regions. Two patients were excluded because they were diagnosed with a pheochromocytoma. Therefore, the study group comprised 124 patients with HNPGL.

Plasma MN levels in HNPGL patients were compared with those of 26 healthy subjects. The subjects who participated in the 'Dietary influences on plasma and urinary MNs' served as a healthy control group. The healthy controls were studied using the same methods as described in our study (e.g. blood was drawn from a forearm vein after an overnight fast; the sample was drawn after $30 \mathrm{~min}$ of rest in the supine position, and furthermore, subjects avoided catecholaminecontaining products). The control group comprised 26 healthy subjects (13 men and 13 women) with a median age of 38 years (9).

The study was an evaluation of routine patient care. According to the requirements of Dutch law, it was not necessary to obtain permission from the Institutional Ethical Commission. Prior to germ line mutation testing, informed consent was obtained from each patient.

\section{Assays}

Free epinephrine, norepinephrine, and dopamine excretion rates in 24-h urine collections were quantified by reversed phase HPLC with electrochemical detection. Inter- and intra-assay coefficients of variation $(\mathrm{CV})$ for epinephrine were $4.3-9.0 \%$ ranging from low to high concentrations. For norepinephrine, these data are $2.7-3.6 \%$ and for dopamine 3.1-4.8\%. Vanillylmandelic acid (VMA) excretion in urine was measured using HPLC with fluorometric detection with inter- and intra-assay CV of 2.4-9.1\%. Urinary deconjugated (nor)metanephrine and 3MT were determined by isotope dilution gas chromatography with mass spectrometric detection. The $\mathrm{CV}$ of the 3-O-methylated catecholamine metabolites (MN, normetanephrine (NMN), and 3MT) ranged from 1.7 to $4.2 \%$ (10). Plasma free MNs were determined by automated in-line solid-phase extraction and isotope dilution liquid chromatography with mass spectrometric detection (11).

Reference ranges were obtained in healthy volunteers. These values were for urinary excretion: free norepinephrine $0.06-0.47 \mu \mathrm{mol} / 24 \mathrm{~h}$, epinephrine $<0.16 \mu \mathrm{mol} / 24 \mathrm{~h}$, dopamine $0.46-3.40 \mu \mathrm{mol} / 24 \mathrm{~h}$, VMA $<30 \mu \mathrm{mol} / 24 \mathrm{~h}$ (deconjugated), MN (deconjugated) 33-99 $\mu \mathrm{mol} / \mathrm{mol}$ creatinine, NMN (deconjugated) 64-260 $\mu \mathrm{mol} / \mathrm{mol}$ creatinine, and 3MT (deconjugated) $45-197 \mu \mathrm{mol} / \mathrm{mol}$ creatinine (12).

The reference intervals for plasma free MNs were determined using blood samples collected in the supine position from 115 volunteers ( 57 males and 58 females; age range, 36-81 years; median age, 55 years) (13). The reference ranges for plasma free $\mathrm{MNs}$ were $\mathrm{MN}$ $0.07-0.33 \mathrm{nmol} / \mathrm{l}$, NMN $0.23-1.07 \mathrm{nmol} / \mathrm{l}$, and $3 \mathrm{MT}$ $<0.17 \mathrm{nmol} / \mathrm{l}$. SDH mutation analysis was performed by restriction digestion as described by Taschner et al. $(14,15)$.

\section{Statistical analysis}

SPSS for Windows version 16.0 (SPSS, Inc.) was used for data analysis. Results are expressed as means \pm s.e.M., unless otherwise specified. Test sensitivity was calculated from the patients with a positive test result divided by the total number of patients tested. Test sensitivities were compared using the McNemar test. The average value of catecholamine and metabolite excretion rates of two urine samples was used for calculation of $P$ values. A $P$ value $<0.05$ was considered to represent a significant difference. 
Table 1 Clinical characteristics of 124 patients with head and neck paragangliomas (HNPGLs). Data are shown as mean \pm s.E.M., unless otherwise indicated.

\begin{tabular}{lccc}
\hline & $\begin{array}{c}\text { All patients } \\
\text { with HNPGL } \\
(n=124)\end{array}$ & $\begin{array}{c}\text { Hereditary } \\
\text { HNPGL } \\
(n=95)\end{array}$ & $\begin{array}{c}\text { Sporadic } \\
\text { HNPGL } \\
(n=16)\end{array}$ \\
\hline Patient characteristics & & & \\
Age (years) & $49 \pm 1$ & $47 \pm 1$ & $58 \pm 3$ \\
Gender $(n(\%))$ & $64(52 \%)$ & $51(54 \%)$ & $4(25 \%)$ \\
$\quad$ Men & $60(48 \%)$ & $44(46 \%)$ & $12(75 \%)$ \\
$\quad$ Women & & & \\
Type of glomus tumor & & & \\
$(n(\%))$ & $87(70 \%)$ & $73(77 \%)$ & $4(25 \%)$ \\
Caroticum & $51(41 \%)$ & $42(44 \%)$ & $6(38 \%)$ \\
Vagale & $13(11 \%)$ & $11(12 \%)$ & $1(6 \%)$ \\
Jugulare & $21(17 \%)$ & $13(14 \%)$ & $5(31 \%)$ \\
Jugulotympanicum & $12(10 \%)$ & $6(6 \%)$ & $5(31 \%)$ \\
Tympanicum & & & $12(75 \%)$ \\
No. of HNPGL $(n(\%))$ & $55(44 \%)$ & $37(39 \%)$ & $4(25 \%)$ \\
1 & $69(56 \%)$ & $58(61 \%)$ & \\
$>1$ & & & \\
No. of patients with & & & \\
$\quad$ genetic analysis & $86(69 \%)$ & & \\
SDHD & $8(7 \%)$ & & \\
SDHB & $1(1 \%)$ & & \\
SDHC & & & \\
Sporadic & $16(13 \%)$ & & \\
\hline
\end{tabular}

\section{Results}

\section{Clinical characteristics}

The study group comprised 124 patients with HNPGL. Mean age of the patients was 49 years (range 13-77 years; Table 1). Fifty-five patients $(44 \%)$ had a single HNPGL, whereas 69 patients $(56 \%)$ had multiple HNPGLs. Paragangliomas occurred most frequently in the glomus caroticum $(70 \%)$. Fifty-one patients $(41 \%)$ had a glomus vagale tumor, $21(17 \%)$ a glomus jugulotympanicum tumor, $13(11 \%)$ a glomus jugulare tumor, and $12(10 \%)$ a glomus tympanicum tumor.

Genetic testing for SDHx mutations was performed in 111 of the 124 patients (90\%) with HNPGL. In 95 patients, mutations were found (86 SDHD, eight SDHB, and one $S D H C$ ), whereas in 16 patients, no mutation was found. In four patients with multiple HNPGLs, no mutation in the $S D H B, S D H C$, or $S D H D$ gene was found. We screened these four patients for a mutation in the SDAF2 gene, but in none of these four patients was a mutation found.

\section{Biochemical characteristics}

Forty-six patients $(37 \%)$ had biochemically active HNPGL, evidenced by increased plasma concentrations of free (nor)metanephrine, 3MT and/or increased 24-h urinary concentrations of free catecholamines, and deconjugated metabolites, including 3MT (Table 2). There was no difference between the number of subjects with increased plasma free 3MT concentrations compared with the number of subjects with increased urinary excretion rates of deconjugated $3 \mathrm{MT}$ ( $n=35$ vs $30,28$ vs $24 \%, P=0.13)$. Urinary deconjugated $\mathrm{MN}$ excretion rates (MN and NMN) were increased in $8 \%$ (10 of 124 patients), which in combination with the patients with increased urinary deconjugated 3MT concentration increased the number of patients with biochemically active HNPGL to 27\% (33 of 124 patients). Eleven patients (9\%) had increased urinary excretion rates of VMA. Increased urinary excretion rates of free catecholamines (adrenaline, noradrenaline, or dopamine) were present in $10 \%$ of the patients (12 of 124 patients). Increased urinary excretion rates of combined MNs in combination with catecholamines were present in $28 \%$ of the patients ( 35 of 124 patients).

Plasma free (nor)metanephrine concentrations were increased in $11 \%$ of the patients ( 14 of 124 patients). The addition of plasma free 3MT levels to these measurements increased the proportion of patients with evidence of biochemically active HNPGL to 33\% (41 of 124 patients). The number of HNPGL patients with increased plasma free MN levels was not significantly different from that of patients with increased 24-h urinary excretion rates of deconjugated MNs (11 vs $8 \%, P=0.34$ ). Increased plasma free $M N$ ( $\mathrm{MN}$ and $\mathrm{NMN}$ ) concentrations in combination with increased plasma free 3MT levels were present in significantly more HNPGL subjects than increased urinary excretion rates of combined MNs ( $\mathrm{MN}$ and NMN) including 3MT (33 vs $27 \%, P<0.05$ ).

In total, ten patients had either increased urinary or increased plasma MN levels. Of these ten patients, four had increased plasma 3MT levels, one had an increased

Table 2 Plasma metanephrine levels and mean urinary excretion rates of catecholamines, VMA, and metanephrines in 124 HNPGL patients.

\begin{tabular}{|c|c|c|}
\hline & $\begin{array}{l}\text { Mean } \\
\text { (range) }\end{array}$ & $\begin{array}{l}\mathrm{N} \text { tested } \\
\text { positive }\end{array}$ \\
\hline \multicolumn{3}{|l|}{ Plasma } \\
\hline NMN (nmol/l) & $0.6(0.16-6.64)$ & $7(6 \%)$ \\
\hline $\mathrm{MN}(\mathrm{nmol} / \mathrm{l})$ & $0.18(0.04-0.48)$ & $7(6 \%)$ \\
\hline 3MT (nmol//l) & $0.46(0.03-12.8)$ & $35(28 \%)$ \\
\hline $\mathrm{MN}+\mathrm{NMN}(\mathrm{nmol} / \mathrm{l})$ & $0.78(0.28-6.78)$ & $14(11 \%)$ \\
\hline $\mathrm{MN}+\mathrm{NMN}+3 \mathrm{MT}(\mathrm{nmol} / \mathrm{l})$ & $1.24(0.32-13.9)$ & $41(33 \%)$ \\
\hline \multicolumn{3}{|l|}{ Urine } \\
\hline NMN ( $\mu \mathrm{mol} / \mathrm{mol}$ creatinine) & $176(55-1720)$ & $5(4 \%)$ \\
\hline $\mathrm{MN}(\mu \mathrm{mol} / \mathrm{mol}$ creatinine $)$ & $55(16-162)$ & $6(5 \%)$ \\
\hline 3MT ( $\mu \mathrm{mol} / \mathrm{mol}$ creatinine $)$ & $312(43-6391)$ & $30(24 \%)$ \\
\hline $\mathrm{MN}+\mathrm{NMN}(\mu \mathrm{mol} / \mathrm{mol}$ creatinine $)$ & $232(95.5-1783)$ & $10(8 \%)$ \\
\hline $\begin{array}{l}\mathrm{MN}+\mathrm{NMN}+3 \mathrm{MT}(\mu \mathrm{mol} / \mathrm{mol} \\
\text { creatinine })\end{array}$ & $544(154-6598)$ & $33(27 \%)^{a}$ \\
\hline Adrenaline $(\mu \mathrm{mol} / 24 \mathrm{~h})$ & $0.02(0.0-0.11)$ & 0 \\
\hline Noradrenaline $(\mu \mathrm{mol} / 24 \mathrm{~h})$ & $0.40(0.11-5.2)$ & $6(5 \%)$ \\
\hline Dopamine $(\mu \mathrm{mol} / 24 \mathrm{~h})$ & $1.92(0.77-6.43)$ & $8(7 \%)$ \\
\hline Catecholamines $(\mu \mathrm{mol} / 24 \mathrm{~h})$ & $2.34(0.91-9.78)$ & $12(10 \%)$ \\
\hline VMA $(\mu \mathrm{mol} / 24 \mathrm{~h})$ & $22.5(0-62)$ & $11(9 \%)$ \\
\hline \multicolumn{2}{|c|}{ Catecholamines + combined MNs } & $35(28 \%)$ \\
\hline
\end{tabular}

VMA, vanillylmandelic acid; MN, metanephrine; NMN, normetanephrine; 3MT, 3-methoxytyramine; combined MNs, MN + NMN + 3MT.

${ }^{\mathrm{a}} \mathrm{A}$ significant difference in test sensitivity between urine and plasma. 
plasma NMN level, and one had increased NMN and 3MT levels. Overall, six patients had additionally increased noradrenergic or dopaminergic activity. Four patients showed no increased noradrenergic or dopaminergic activity.

Eight patients had increased urinary excretion rates of dopamine. Seven of these eight patients had increased plasma 3MT levels and the other patient had an increased plasma NMN level.

Nine patients tested negative with urinary screening of catecholamine excretion rates but positive with screening of plasma MN levels. Of these patients, six had increased plasma free 3MT levels, two had increased plasma free $\mathrm{MN}$ levels, and one had an increased plasma free NMN level.

In the healthy control group, two persons had slightly elevated MN levels, none had increased plasma free NMN levels, and none had increased plasma 3MT levels. We have presented the results of the plasma free MN measurements graphically in Fig. 1.

\section{Catecholamine and metabolite excretion, tumor load, and genotype}

We identified 55 patients with only a single HNPGL with a mean tumor diameter of $3.3 \mathrm{~cm}$ (range $0.3-9.7 \mathrm{~cm}$ ). In those patients, there was a weak positive correlation between tumor diameters and plasma concentrations of free 3MT ( $r=0.30, P=0.04)$, and the combined plasma concentrations of free MN, NMN, and 3MT $(r=0.35$, $P=0.02$ ). There were also weak correlations between tumor diameters and mean urinary excretion rates of deconjugated NMN $(r=0.40, P<0.01)$, VMA $(r=0.32$, $P=0.03)$, dopamine $(r=0.40, P<0.01)$, the combined excretion rates of deconjugated $\mathrm{MN}$ and $\mathrm{NMN}(r=0.40$, $P<0.01)$, the combined excretion rates of deconjugated (nor)metanephrine and 3MT $(r=0.43, P<0.01)$, and the mean excretion rate of catecholamines $(r=0.37$,
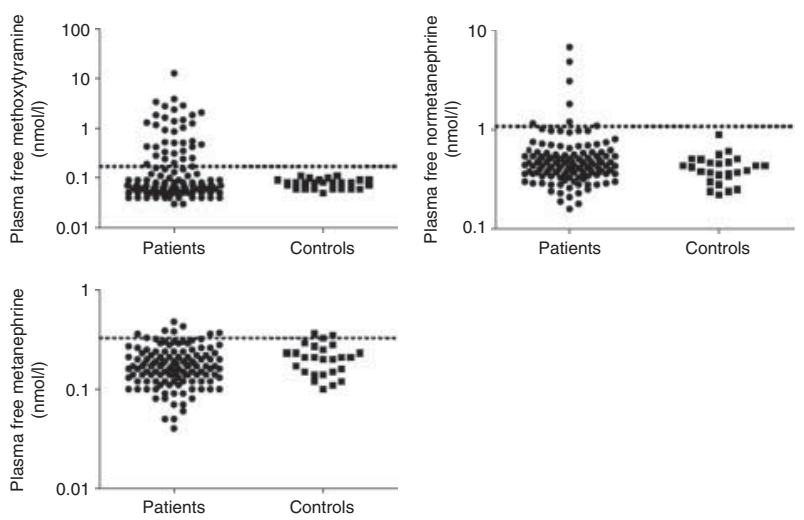

Figure 1 Plasma concentrations of free methoxytyramine, normetanephrine, and metanephrine in HNPGL patients (left) and controls (right). The dashed line represents the upper limit of the reference interval.
$P=0.01)$. There was no correlation between the number of HNPGL and plasma free MN levels and mean urinary excretion rates of deconjugated MNs and catecholamines.

We found no specific biochemical phenotype for patients with either a $S D H B$ or a $S D H D$ genotype. The number of patients with a biochemically active tumor was equal between patients with a SDHB, a SDHD, or a sporadic genotype.

\section{Catecholamine and metabolite excretion, age, BMI, and gender}

Stepwise linear regression analysis was performed in a model including age, gender, and BMI as independent variables and plasma free $\mathrm{MN}, \mathrm{NMN}$, and 3MT levels as dependant variables. We identified age $(\beta=0.35$, $P<0.001)$, BMI $(\beta=-0.29, P<0.001)$, and gender $(\beta=-0.35, P<0.001)$ as independent predictors of plasma free MN levels. The patients with increased plasma free MN levels were significantly older compared with patients with normal levels $(48.0 \pm 1.2$ vs 61.5 \pm 3.7 years, $P<0.01)$. Men had significantly higher plasma free MN levels compared with women $(0.21$ \pm 0.0 vs $0.15 \pm 0.0, P<0.001)$.

There was no relationship of plasma levels of free NMN with age $(\beta=0.09, P=0.35)$, BMI $(\beta=-0.11$, $P=0.26)$, and gender $(\beta=0.09, P=0.35)$. There was a relationship of age $(\beta=0.09, P=0.38)$, BMI $(\beta=0.02, P=0.41)$, and gender $(\beta=-0.08, P=0.41)$ with plasma free 3MT levels.

\section{Long-term follow-up of patients with biochemically active tumor}

Forty-six patients $(37 \%)$ had biochemically active HNPGL, evidenced by increased plasma concentrations of free (nor)metanephrine, 3MT and/or increased 24-h urinary concentrations of free catecholamines, and deconjugated metabolites, including 3MT. In this cohort of 46 patients, mean follow-up time was $2.8 \pm 1.1$ years, with a total follow-up time of 129 patient years. Forty patients had persistent catecholamine excess during follow-up. Five patients had normalization of catecholamine levels, of whom two patients underwent surgery. Three patients showed normalization of catecholamine excretion levels without intervention (surgery) and one patient was lost to follow-up.

Thirty-eight patients had persistent catecholamine excess during follow-up. Three patients had normalization of catecholamine levels, of whom two underwent surgery. In four patients, either one of the two urine samples showed increased MN levels. As we consider a patient positive when two urine samples contain excess catecholamines, these patients were considered to have normalization of catecholamine excretion. One patient was lost to follow-up. 


\section{Discussion}

The results of this study show that one-third of HNPGL patients have biochemically active tumors. The combined plasma concentrations of free MNs and 3MT indicate a higher proportion of patients with biochemically active HNPGL than 24-h urinary excretion rates of deconjugated MNs and 3MT. In addition, the current data indicate that in HNPGL patients, plasma free 3MT concentrations and urinary excretion rates of deconjugated 3MT do not identify significantly different numbers of subjects with biochemically active HNPGL. Plasma MN levels were additionally measured in a healthy control group. In these controls, two persons had slightly elevated MN levels, none had increased plasma free NMN levels, and none had increased plasma 3MT levels.

HNPGLs have the ability to produce and secrete catecholamines $(2,3)$. Biochemically active paragangliomas are identified by the measurement of plasma (nor)metanephrine and 3MT concentrations and 24-h urinary excretion rates of free catecholamines and their deconjugated metabolites $(6,16)$. The measurements of urinary excretion rates of catecholamine metabolites (i.e. MN and NMN) and especially plasma levels of catecholamines and their metabolites are recommended for the biochemical screening of pheochromocytoma because of their high diagnostic sensitivity and specificity (7). However, the majority of patients with biochemically active HNPGL secrete 3MT, which is a metabolite of dopamine. Therefore, the measurement of this metabolite should be included if biochemical activity of HNPGL in general is assessed. 3MT can be measured in urine and plasma by HPLC-tandem mass spectrometric detection (XLC-MS/MS) (13). This study indicates that the assessment of plasma free 3MT levels does not add to the measurement of urinary excretion rates of deconjugated 3MT.

As patients with HNPGL have the ability to (co)secrete noradrenaline $(11,17,18)$, this catecholamine and its metabolite NMN should be measured as well. Combining the results of free MNs and 3MT in plasma resulted in a slightly, but significantly, higher number of patients with biochemically active HNPGL compared with the combined results of urinary excretion rates.

Consumption of catecholamine-rich food products can result in substantial increases in urinary excretion rates of deconjugated NMN and 3MT and to a lesser extent in plasma free 3MT levels. Therefore, dietary restrictions are indicated prior to collection of blood for measurements of plasma free 3MT levels, and urinary excretion rates of deconjugated NMN and 3MT (9). Although patients in our study collected urine during $48 \mathrm{~h}$ under strict dietary regulations, we cannot exclude potential confounding effects of the diet. In contrast, the measurements of plasma free $\mathrm{MN}$ concentrations are not influenced by the confounding effects of dietary components; this contributes to the highest sensitivity of plasma levels compared with the measurement of urinary excretion rates of $\mathrm{MN}(9,19)$. In accordance, plasma free MNs (MN, NMN, and 3MT) levels were increased in a higher percentage of HNPGL patients compared with urinary excretion rates of MNs.

Our findings are in agreement with the observations in patients with pheochromocytomas $(6,7,8)$. Lenders et al. reported a test sensitivity of $97 \%$ of plasma free MNs vs only $60 \%$ of urinary combined MNs in patients with hereditary pheochromocytomas and 99\% of plasma MN levels vs $88 \%$ in urinary excretion rates in patients with sporadic pheochromocytomas.

HNPGLs are devoid of phenylethanolamine $N$-methyltransferase enzymatic activity. The increase in urinary MN levels observed in patients with HNPGL is therefore somewhat peculiar. We identified age, BMI, and gender as independent predictors of plasma MN levels. Patients with increased plasma free MN levels were significantly older compared with those with normal free plasma MN levels. For the levels of plasma free NMN and 3MT, we did not find a relationship between plasma and/or urine levels and age, gender, and BMI. Deutschbein et al. found that MN levels were significantly influenced by sex and age. Our data are therefore in correspondence with the data of Deutschbein et al. (20). They also found a relationship between plasma NMN levels and BMI and sex. We did not find these relationships. As HNPGLs have the ability to secrete noradrenaline, but not adrenaline, the presence of HNPGLs could influence the correlation between plasma NMN levels, age, sex, and BMI. Because in all patients with increased catecholamine/MN levels additional radiological assessment excluded the presence of a pheochromocytoma, the increased levels of MN may be regarded as physiological. It could indicate that men and older people have increased adrenergic activity and/or the removal of $\mathrm{MN}$ spillover from the adrenals may be less adequate compared with younger people. The results of the (nor)metanephrine measurements in healthy subjects strengthen our assumption. As none of the healthy subjects had increased NMN and 3MT levels, but two had increased MN levels, the excretion of MN could be considered as physiological.

Medication (tricyclic antidepressants, $\beta$-blockers, calcium antagonists, and acetaminophen) can either cause direct analytical interference in measurements of catecholamines and metabolite levels or may influence the physiological processes that determine these levels. Therefore, urine and plasma samples are collected after withdrawal of medication for several weeks or after changing antihypertensive medication to doxazosin. In total, 16 patients used doxazosin because of hypertension during the study period. Eisenhofer et al. (21) studied the effect of medication-associated false-positive test results in patients screened for pheochromocytoma. They found that selective $\alpha 1$-blockers were not associated with an increased frequency of false-positive results for plasma norepinephrine or NMN but were associated with false-positive elevations of 
urinary norepinephrine. Because of these findings, we do not expect that doxazosin caused an increase in either plasma or urinary NMN levels.

In conclusion, one-third of HNPGL patients have biochemically active tumors. The combined assessment of plasma concentrations of free MNs and 3MT detects a higher number of biochemically active HNPGLs than the measurement of 24-h urinary excretion rates of combined MNs and 3MT. In addition, the current data indicate that in HNPGL patients, urinary excretion rates of deconjugated 3MT and plasma free 3MT levels do not identify significantly different numbers of subjects with biochemically active HNPGL.

\section{Declaration of interest}

The authors declare that there is no conflict of interest that could be perceived as prejudicing the impartiality of the research reported.

\section{Funding}

This research did not receive any specific grant from any funding agency in the public, commercial or not-for-profit sector.

\section{References}

1 DeLellis RA, Lloyd RV, Heitz PU \& Eng C. In World Health Organisation Classification of Tumors, Pathology and Genetics of Endocrine Organs. Lyon: IARC Press, 2004.

2 Erickson D, Kudva YC, Ebersold MJ, Thompson GB, Grant CS, van Heerden JA \& Young WF Jr. Benign paragangliomas: clinical presentation and treatment outcomes in 236 patients. Journal of Clinical Endocrinology and Metabolism 200186 5210-5216. (doi:10.1210/jc.86.11.5210)

3 van Duinen N, Steenvoorden D, Kema IP, Jansen JC, Vriends AH, Bayley JP, Smit JW, Romijn JA \& Corssmit EP. Increased urinary excretion of 3-methoxytyramine in patients with head and neck paragangliomas. Journal of Clinical Endocrinology and Metabolism 201095 209-214. (doi:10.1210/jc.2009-1632)

4 Zak FG \& Lawson W. In The Paraganglionic Chemoreceptor System: Physiology, Pathology and Clinical Medicine, 1st edn. New York: Springer-Verlag, Inc., 1982.

5 Eisenhofer G, Lenders JW, Linehan WM, Walther MM, Goldstein DS \& Keiser HR. Plasma normetanephrine and metanephrine for detecting pheochromocytoma in von Hippel-Lindau disease and multiple endocrine neoplasia type 2. New England Journal of Medicine 1999b 340 1872-1879. (doi:10.1056/NEJM199906173402404)

6 Lenders JW, Keiser HR, Goldstein DS, Willemsen JJ, Friberg P, Jacobs MC, Kloppenborg PW, Thien T \& Eisenhofer G. Plasma metanephrines in the diagnosis of pheochromocytoma. Annals of Internal Medicine 1995123 101-109. (doi:10.7326/0003-4819123-2-199507150-00004)

7 Lenders JW, Pacak K, Walther MM, Linehan WM, Mannelli M, Friberg P, Keiser HR, Goldstein DS \& Eisenhofer G. Biochemical diagnosis of pheochromocytoma: which test is best? Journal of the American Medical Association 2002287 1427-1434. (doi:10.1001/ jama.287.11.1427)

8 Sawka AM, Jaeschke R, Singh RJ \& Young WF Jr. A comparison of biochemical tests for pheochromocytoma: measurement of fractionated plasma metanephrines compared with the combination of 24-hour urinary metanephrines and catecholamines. Journal of Clinical Endocrinology and Metabolism 2003 88 553-558. (doi:10.1210/jc.2002-021251)

9 de Jong WH, Eisenhofer G, Post WJ, Muskiet FA, de Vries EG \& Kema IP. Dietary influences on plasma and urinary metanephrines: implications for diagnosis of catecholamineproducing tumors. Journal of Clinical Endocrinology and Metabolism 200994 2841-2849. (doi:10.1210/jc.2009-0303)

10 Kema IP, Meiborg G, Nagel GT, Stob GJ \& Muskiet FA. Isotope dilution ammonia chemical ionization mass fragmentographic analysis of urinary 3-O-methylated catecholamine metabolites. Rapid sample clean-up by derivatization and extraction of lyophilized samples. Journal of Chromatography 1993617 181-189.

11 Hall FT, Perez-Ordonez B, Mackenzie RG \& Gilbert RW. Does catecholamine secretion from head and neck paragangliomas respond to radiotherapy? Case report and literature review. Skull Base 200313 229-234. (doi:10.1055/s-2004-817699)

12 Willemsen JJ, Ross HA, Wolthers BG, Sweep CG \& Kema IP. Evaluation of specific high-performance liquid-chromatographic determinations of urinary metanephrine and normetanephrine by comparison with isotope dilution mass spectrometry. Annals of Clinical Biochemistry 200138 722-730. (doi:10.1258/ 0004563011900984)

13 de Jong WH, Graham KS, van der Molen JC, Links TP, Morris MR, Ross HA, de Vries EG \& Kema IP. Plasma free metanephrine measurement using automated online solid-phase extraction HPLC tandem mass spectrometry. Clinical Chemistry $2007 \mathbf{5 3}$ 1684-1693. (doi:10.1373/clinchem.2007.087114)

14 Bayley JP, van Minderhout I, Weiss MM, Jansen JC, Oomen PH, Menko FH, Pasini B, Ferrando B, Wong N, Alpert LC et al. Mutation analysis of SDHB and SDHC: novel germline mutations in sporadic head and neck paraganglioma and familial paraganglioma and/or pheochromocytoma. BMC Medical Genetics 20067 1. (doi:10.1186/1471-2350-7-1)

15 Taschner PE, Jansen JC, Baysal BE, Bosch A, Rosenberg EH, Brocker-Vriends AH, van Der Mey AG, van Ommen GJ, Cornelisse CJ \& Devilee P. Nearly all hereditary paragangliomas in the Netherlands are caused by two founder mutations in the SDHD gene. Genes, Chromosomes \& Cancer 200131 274-281. (doi:10.1002/gcc.1144)

16 Eisenhofer G, Goldstein DS, Sullivan P, Csako G, Brouwers FM, Lai EW, Adams KT \& Pacak K. Biochemical and clinical manifestations of dopamine-producing paragangliomas: utility of plasma methoxytyramine. Journal of Clinical Endocrinology and Metabolism 200590 2068-2075. (doi:10.1210/jc.2004-2025)

17 Havekes B, van der Klaauw AA, Weiss MM, Jansen JC, van Der Mey AG, Vriends AH, Bonsing BA, Romijn JA \& Corssmit EP. Pheochromocytomas and extra-adrenal paragangliomas detected by screening in patients with SDHD-associated head-and-neck paragangliomas. Endocrine-Related Cancer 200916 527-536. (doi:10.1677/ERC-09-0024)

18 van Houtum WH, Corssmit EP, Douwes Dekker PB, Jansen JC, van Der Mey AG, Brocker-Vriends AH, Taschner PE, Losekoot M, Frolich M, Stokkel MP et al. Increased prevalence of catecholamine excess and phaeochromocytomas in a well-defined Dutch population with SDHD-linked head and neck paragangliomas. European Journal of Endocrinology 2005152 87-94. (doi:10.1530/ eje.1.01833)

19 Eisenhofer G, Coughtrie MW \& Goldstein DS. Dopamine sulphate: an enigma resolved. Clinical and Experimental Pharmacology $\mathcal{E}$ Physiology. Supplement 1999a 26 S41-S53.

20 Deutschbein T, Unger N, Jaeger A, Broecker-Preuss M, Mann K \& Petersenn S. Influence of various confounding variables and storage conditions on metanephrine and normetanephrine levels in plasma. Clinical Endocrinology 201073 153-160. (doi:10.111/ j.1365-2265.2009.03761.x)

21 Eisenhofer G, Goldstein DS, Walther MM, Friberg P, Lenders JW, Keiser HR \& Pacak K. Biochemical diagnosis of pheochromocytoma: how to distinguish true- from false-positive test results. Journal of Clinical Endocrinology and Metabolism 200388 2656-2666. (doi:10.1210/jc.2002-030005)

Received 27 June 2013

Accepted 4 July 2013 